\title{
Water-wave scattering by an ice-strip
}

\author{
RUPANWITA GAYEN (CHOWDHURY), B.N. MANDAL and \\ A. CHAKRABARTI ${ }^{1}$ \\ Physics and Applied Mathematics Unit, Indian Statistical Institute, 203, B.T. Road, Kolkata 700 108, India \\ (biren@isical.ac.in); ${ }^{1}$ Department of Mathematics, Indian Institute of Science, Bangalore 560012, India
}

\begin{abstract}
The problem of water-wave scattering by a strip of ice-cover floating on the surface of deep water is investigated here. The ice-cover is modelled as a thin elastic plate of very small thickness. The problem is reduced to that of solving two singular integral equations of Carleman type over a semi-infinite range and are solved approximately by casting them into two separate Riemann-Hilbert problems by assuming the strip width to be large. The reflection and transmission coefficients are derived approximately. Numerical results for the reflection coefficient are presented graphically against the wave number and also against the ice-cover parameter. The oscillatory nature of the reflection coefficient against the wave number as well as the ice-cover parameter is found to be one of the main features of the curves. It is also seen that, in the limiting case when the ice-cover parameter tends to zero (i.e., the ice-cover is almost absent), the amount of reflection is negligible.
\end{abstract}

Key words: Carleman integral equation, ice-strip, reflection coefficient, water-wave scattering

\section{Introduction}

The scattering of surface water waves by several discontinuities has been a problem of long-standing interest. If some part of the surface of water is covered by ice and the remaining part is free, then discontinuities occur in the surface boundary conditions. Again, the ice may be classified into two categories. In the first category it is modelled as an inertial surface composed of discrete particles having no elasticity. Another classification is to model the ice-sheet as a thin elastic plate. In that case the related scattering problem is very important because of its application in the construction of very large floating structures and in ice-wave interaction problems in polar regions.

Evans and Davies [1] considered the problem of scattering of obliquely incident waves on a semi-infinite thin elastic plate in water of finite depth. They used the Wiener-Hopf technique to obtain an exact solution of the problem. However, the solution was too complicated for obtaining numerical results. This has been overcome recently by Chung and Fox [2] using some efficient algebraic manipulations. Gol'dshtein and Marchenko [3] and Tkacheva [4-6] also used the Wiener-Hopf technique to study some related scattering problems involving deep, as well as finite-depth water. Earlier on, Fox and Squire [7] used a matching technique where matching was achieved by minimizing a certain error integral evaluated at the point of discontinuity on the upper surface of water to obtain the reflection and transmission coefficients for the problem originally considered by Evans and Davies [1]. The residue-calculus technique is a somewhat simple alternative method compared to the difficult Wiener-Hopf technique, which has been successfully applied in the water-wave literature by Linton [8], Linton and Chung [9], Chung and Linton [10] to investigate wave-scattering problems involving a rigid dock as well as ice-sheets. Hermans $[11,12]$ recently used a method 
based on solving an integral equation along the region of the water surface which contains a floating dock or floating ice (thin elastic plate), by means of a superposition of exponential functions. This method is somehow related to the residue-calculus technique. Two-dimensional scattering of normally incident surface water waves in deep water was considered by Chakrabarti $[13,14]$ wherein a point of discontinuity arises due to two semi-infinite inertial surfaces and a semi-infinite ice-cover floating on clean water, respectively. Both problems were solved exactly by reducing them to Carleman-type singular integral equations over a semiinfinite range. The physical quantities of interest, namely the reflection and the transmission coefficients, were then derived in explicit forms.

If the ice-cover is in the form of a finite strip instead of a semi-infinite one, explicit solutions are no longer possible to obtain. In the present paper we consider a thin sheet of ice of finite width, but infinitely long in its spread and is floating on the surface of deep water. The problem is formulated in terms of appropriate velocity potentials in the regions below the free surface and below the strip. Using Havelock's inversion theorem we reduce it to two coupled singular integral equations of the Carleman type. Assuming the width of the strip to be large, these equations are decoupled and then solved approximately by converting them into two Riemann-Hilbert problems. We call the solutions to the decoupled equations the zero-order solutions and use them in the coupled integral equations and again solve them for the first-order approximate solutions by a similar method. This is a sort of iteration process and, in principle, can be applied to obtain higher-order iterative solutions. But we confine ourselves to first-order solutions only, since the first-order approximations to the reflection and transmission coefficients yield results that are quite satisfactory. The numerical results for the reflection coefficient so obtained are presented graphically in a number of figures. Multiple reflections have been noticed to be the key feature of every figure. Making the ice-cover parameter very small, it is observed that the reflection coefficient becomes almost zero, as expected.

\section{Mathematical formulation of the problem}

A two-dimensional rectangular Cartesian co-ordinate system $(x, y)$ is chosen in which the $y$-axis is taken vertically downwards into the water which is homogeneous with constant density $\rho$ and inviscid. Suppose a strip of ice-cover of width $l$ occupies the region $y=0,0<$ $x<l$. The strip is assumed to be infinitely long along the horizontal $z$-direction. Considering two-dimensional and time-harmonic motion with angular frequency $\sigma$ and linear theory, if $\Phi(x, y, t)=\mathfrak{R}\left\{\left\{(x, y) \mathrm{e}^{-\mathrm{i} \sigma t}\right\}\right.$ denotes the velocity potential describing the motion, we have that $\phi$ satisfies the Laplace's equation

$$
\nabla^{2} \phi=0, \quad y \geq 0, \quad-\infty<x<\infty,
$$

the free-surface condition

$$
K \phi+\phi_{y}=0 \text { on } y=0,-\infty<x<0 \text { and } x>l
$$

and the condition on the ice-cover

$$
K \phi+\phi_{y}+D \phi_{y x x x x}=0 \text { on } y=0, \quad 0<x<l,
$$

where $D=\frac{E h^{3}}{12\left(1-\nu^{2}\right) \rho g} ; E, v$ are, respectively, Young's modulus and Poisson's ratio of ice, $g$ is the acceleration due to gravity, $h$ is the very small thickness of ice of which a still smaller part is immersed in water. The form of the ice-cover condition is derived on the assumption 
that the waves are long compared to the thickness of the ice-strip $([3,14])$. However, this condition is not necessary in the derivation of the ice-cover condition in the form (2.3). This is briefly explained in the Appendix A.

Also $\phi$ satisfies the bottom condition

$$
\nabla \phi \rightarrow 0 \quad \text { as } y \rightarrow \infty
$$

and the radiation conditions

$$
\phi \rightarrow \begin{cases}\mathrm{e}^{-K y+\mathrm{i} K x}+R \mathrm{e}^{-K y-\mathrm{i} K x} & \text { as } x \rightarrow-\infty, \\ T \mathrm{e}^{-K y+\mathrm{i} K(x-l)} & \text { as } x \rightarrow \infty,\end{cases}
$$

where $R$ and $T$ are the unknown reflection and transmission coefficients to be determined. Due to zero bending moment and zero shear stress at the two ends of the ice-strip, we have the edge conditions

$$
\phi_{y x x} \rightarrow 0 \quad \text { as } x \rightarrow 0+0, \quad l-0, \quad y=0
$$

and

$$
\phi_{y x x x} \rightarrow 0 \quad \text { as } x \rightarrow 0+0, \quad l-0, \quad y=0 .
$$

Also in the strip region,

$$
\phi(x, y)=\alpha \mathrm{e}^{-\lambda K y+\mathrm{i} \lambda K x}+\beta \mathrm{e}^{-\lambda K y-\mathrm{i} \lambda K(x-l)}+\chi(x, y), \quad 0<x<l
$$

where $\alpha$ and $\beta$ are unknown constants and $\chi(x, y)$ is a non-wavy solution of the Laplace equation. The parameters $\beta$ and $\alpha$ may be regarded as the reflection and the transmission coefficients, respectively, through the points $(0, l)$ and $(0,0) ; \lambda K$ is the real positive root of the equation

$$
D k^{5}+k=K
$$

whose other roots are $\left(\lambda_{1} K, \bar{\lambda}_{1} K\right),\left(\lambda_{2} K, \bar{\lambda}_{2} K\right)$ with $\mathfrak{R e}\left(\lambda_{1}\right)>0, \mathfrak{R e}\left(\lambda_{2}\right)<0, \mathfrak{I m}\left(\lambda_{1}, \lambda_{2}\right)>0$.

Let $\psi(x, y)$ be defined by $\phi(x, y)=\psi_{x x}(x, y)$, and be represented by $\psi_{1}, \psi_{2}, \psi_{3}$ in the regions $x<0,0<x<l$ and $x>l(y>0)$, respectively. Then by the Havelock expansion, $\psi_{i}(i=$ $1,2,3)$ can be expressed as

$$
\begin{aligned}
\psi_{1}(x, y)= & -\frac{1}{K^{2}} \mathrm{e}^{-K y+\mathrm{i} K x}-\frac{R}{K^{2}} \mathrm{e}^{-K y-\mathrm{i} K x}+\frac{2}{\pi} \int_{0}^{\infty} \frac{A(\xi) L(\xi, y) \mathrm{e}^{\xi x}}{\xi^{2}+K^{2}} \mathrm{~d} \xi, \quad x<0, \quad y>0 \\
\psi_{2}(x, y)= & -\frac{1}{\lambda^{2} K^{2}}\left\{\alpha \mathrm{e}^{-\lambda K y+\mathrm{i} \lambda K x}+\beta \mathrm{e}^{-\lambda K y-\mathrm{i} \lambda K(x-l)}\right\}-\frac{1}{\lambda_{1}^{2} K^{2}}\left\{A_{1} \mathrm{e}^{-\lambda_{1} K y+\mathrm{i} \lambda_{1} K x}\right. \\
& \left.+A_{2} \mathrm{e}^{-\lambda_{1} K y-\mathrm{i} \lambda_{1} K(x-l)}\right\}-\frac{1}{\lambda_{1}^{2} K^{2}}\left\{A_{3} \mathrm{e}^{-\bar{\lambda}_{1} K y+\mathrm{i} \bar{\lambda}_{1} K(x-l)}+A_{4} \mathrm{e}^{-\lambda_{1} K y-\mathrm{i} \bar{\lambda}_{1} K x}\right\} \\
& +\frac{2}{\pi} \int_{0}^{\infty} \frac{B(\xi) \mathrm{e}^{\xi(x-l)}+C(\xi) \mathrm{e}^{-\xi x}}{P(\xi)} M(\xi, y) \mathrm{d} \xi, \quad 0<x<l, \quad y>0 \\
\psi_{3}(x, y)= & -\frac{T}{K^{2}} \mathrm{e}^{-K y+\mathrm{i} K(x-l)}+\frac{2}{\pi} \int_{0}^{\infty} \frac{G(\xi) L(\xi, y)}{\xi^{2}+K^{2}} \mathrm{e}^{-\xi(x-l)} \mathrm{d} \xi, x>l, y>0
\end{aligned}
$$


where $A_{1}, A_{2}, A_{3}, A_{4}$ are unknown constants and $A(\xi), B(\xi), C(\xi)$ and $G(\xi)$ are unknown functions of suitable order and

$$
\begin{aligned}
& L(\xi, y)=\xi \cos \xi y-K \sin \xi y, \\
& M(\xi, y)=\xi\left(D \xi^{4}+1\right) \cos \xi y-K \sin \xi y
\end{aligned}
$$

and

$$
P(\xi)=\xi^{2}\left(D \xi^{4}+1\right)^{2}+K^{2} .
$$

By utilizing the conditions of continuity of $\psi$ and $\psi_{x}$ across the lines $x=0$ and $x=l(y>0)$ we obtain four relations involving the four unknown functions $A, B, C$ and $G$ which also involve the unknown constants $A_{1}, A_{2}$ etc., and, to these four relations, if we then use Havelock's inversion theorem [15] we obtain the following eight relations in a straight forward manner. (Note that for each application of Havelock's inversion theorem, two distinct relations occur, one of which represents the inversion formula and the other the constraint in the expansion formula of Havelock (see [15])):

$$
\begin{aligned}
& A(\xi)=-\frac{K \xi(\lambda-1)\left(\alpha+\beta \mathrm{e}^{\mathrm{i} \lambda K l}\right)}{\lambda^{2} K^{2}\left(\xi^{2}+\lambda^{2} K^{2}\right)}-\frac{K \xi\left(\lambda_{1}-1\right)\left(A_{1}+A_{2} \mathrm{e}^{\mathrm{i} \lambda_{1} K l}\right)}{\lambda_{1}^{2} K^{2}\left(\xi^{2}+\lambda_{1}^{2} K^{2}\right)} \\
& -\frac{K \xi\left(\bar{\lambda}_{1}-1\right)\left(A_{3} \mathrm{e}^{-\mathrm{i} \bar{\lambda}_{1} K l}+A_{4}\right)}{\bar{\lambda}_{1}^{2} K^{2}\left(\xi^{2}+\bar{\lambda}_{1}^{2} K^{2}\right)}+\frac{\xi^{2}\left(1+D \xi^{4}\right)+K^{2}}{P(\xi)}\left\{B(\xi) \mathrm{e}^{-\xi l}+C(\xi)\right\} \\
& +\frac{2 D K \xi}{\pi} \int_{0}^{\infty} \frac{u^{5}\left\{B(u) \mathrm{e}^{-u l}+C(u)\right\}}{P(u)\left(u^{2}-\xi^{2}\right)} \mathrm{d} u, \\
& \xi A(\xi)=-\frac{\mathrm{i} K \xi(\lambda-1)\left(\alpha-\beta \mathrm{e}^{\mathrm{i} \lambda K l}\right)}{\lambda K\left(\xi^{2}+\lambda^{2} K^{2}\right)}-\frac{\mathrm{i} K \xi\left(\lambda_{1}-1\right)\left(A_{1}-A_{2} \mathrm{e}^{\mathrm{i} \lambda_{1} K l}\right)}{\lambda_{1} K\left(\xi^{2}+\lambda_{1}^{2} K^{2}\right)} \\
& -\frac{\mathrm{i} K \xi\left(\bar{\lambda}_{1}-1\right)\left(A_{3} \mathrm{e}^{-\mathrm{i} \bar{\lambda}_{1} K l}-A_{4}\right)}{\bar{\lambda}_{1} K\left(\xi^{2}+\bar{\lambda}_{1}^{2} K^{2}\right)}+\frac{\xi^{2}\left(1+D \xi^{4}\right)+K^{2}}{P(\xi)} \xi\left\{B(\xi) \mathrm{e}^{-\xi l}-C(\xi)\right\} \\
& +\frac{2 D K \xi}{\pi} \int_{0}^{\infty} \frac{u^{6}\left\{B(u) \mathrm{e}^{-u l}-C(u)\right\}}{P(u)\left(u^{2}-\xi^{2}\right)} \mathrm{d} u, \\
& G(\xi)=-\frac{K \xi(\lambda-1)\left(\alpha \mathrm{e}^{\mathrm{i} \lambda K l}+\beta\right)}{\lambda^{2} K^{2}\left(\xi^{2}+\lambda^{2} K^{2}\right)}-\frac{K \xi\left(\lambda_{1}-1\right)\left(A_{1} \mathrm{e}^{\mathrm{i} \lambda_{1} K l}+A_{2}\right)}{\lambda_{1}^{2} K^{2}\left(\xi^{2}+\lambda_{1}^{2} K^{2}\right)} \\
& -\frac{K \xi\left(\bar{\lambda}_{1}-1\right)\left(A_{3}+A_{4} \mathrm{e}^{-\mathrm{i} \bar{\lambda}_{1} K l}\right)}{\bar{\lambda}_{1}^{2} K^{2}\left(\xi^{2}+\bar{\lambda}_{1}^{2} K^{2}\right)}+\frac{\xi^{2}\left(1+D \xi^{4}\right)+K^{2}}{P(\xi)}\left\{B(\xi)+C(\xi) \mathrm{e}^{-\xi l}\right\} \\
& +\frac{2 D K \xi}{\pi} \int_{0}^{\infty} \frac{u^{5}\left\{B(u)+C(u) \mathrm{e}^{-u l}\right\}}{P(u)\left(u^{2}-\xi^{2}\right)} \mathrm{d} u, \\
& \xi G(\xi)=\frac{\mathrm{i} K \xi(\lambda-1)\left(\alpha \mathrm{e}^{\mathrm{i} \lambda K l}-\beta\right)}{\lambda K\left(\xi^{2}+\lambda^{2} K^{2}\right)}+\frac{\mathrm{i} K \xi\left(\lambda_{1}-1\right)\left(A_{1} \mathrm{e}^{\mathrm{i} \lambda_{1} K l}-A_{2}\right)}{\lambda_{1} K\left(\xi^{2}+\lambda_{1}^{2} K^{2}\right)} \\
& +\frac{\mathrm{i} K \xi\left(\bar{\lambda}_{1}-1\right)\left(A_{3}-A_{4} \mathrm{e}^{-\mathrm{i} \bar{\lambda}_{1} K l}\right)}{\bar{\lambda}_{1} K\left(\xi^{2}+\bar{\lambda}_{1}^{2} K^{2}\right)}-\frac{\xi^{2}\left(1+D \xi^{4}\right)+K^{2}}{P(\xi)} \xi\left\{B(\xi)-C(\xi) \mathrm{e}^{-\xi l}\right\} \\
& -\frac{2 D K \xi}{\pi} \int_{0}^{\infty} \frac{u^{6}\left\{B(u)-C(u) \mathrm{e}^{-u l}\right\}}{P(u)\left(u^{2}-\xi^{2}\right)} \mathrm{d} u,
\end{aligned}
$$




$$
\begin{aligned}
& \frac{1+R}{2}=\frac{\alpha+\beta \mathrm{e}^{\mathrm{i} \lambda K l}}{\lambda^{2}(\lambda+1)}+\frac{A_{1}+A_{2} \mathrm{e}^{\mathrm{i} \lambda_{1} K l}}{\lambda_{1}^{2}\left(\lambda_{1}+1\right)}+\frac{A_{3} \mathrm{e}^{-\mathrm{i} \bar{\lambda}_{1} K l}+A_{4}}{\bar{\lambda}_{1}^{2}\left(\bar{\lambda}_{1}+1\right)}-\frac{2}{\pi} \int_{0}^{\infty} \frac{B(\xi) \mathrm{e}^{-\xi l}+C(\xi)}{Q(\xi)} D K^{4} \xi^{5} \mathrm{~d} \xi, \\
& \frac{1-R}{2}=\frac{\alpha-\beta \mathrm{e}^{\mathrm{i} \lambda K l}}{\lambda(\lambda+1)}+\frac{A_{1}-A_{2} \mathrm{e}^{\mathrm{i} \lambda_{1} K l}}{\lambda_{1}\left(\lambda_{1}+1\right)}+\frac{A_{3} \mathrm{e}^{-\mathrm{i} \bar{\lambda}_{1} K l}-A_{4}}{\bar{\lambda}_{1}\left(\bar{\lambda}_{1}+1\right)}+\frac{2 \mathrm{i}}{\pi} \int_{0}^{\infty} \frac{B(\xi) \mathrm{e}^{-\xi l}-C(\xi)}{Q(\xi)} D K^{3} \xi^{6} \mathrm{~d} \xi, \\
& \frac{T}{2}=\frac{\alpha \mathrm{e}^{\mathrm{i} \lambda K l}+\beta}{\lambda^{2}(\lambda+1)}+\frac{A_{1} \mathrm{e}^{\mathrm{i} \lambda_{1} K l}+A_{2}}{\lambda_{1}^{2}\left(\lambda_{1}+1\right)}+\frac{A_{3}+A_{4} \mathrm{e}^{-\mathrm{i} \bar{\lambda}_{1} K l}}{\bar{\lambda}_{1}^{2}\left(\bar{\lambda}_{1}+1\right)}-\frac{2}{\pi} \int_{0}^{\infty} \frac{B(\xi)+C(\xi) \mathrm{e}^{-\xi l}}{Q(\xi)} D K^{4} \xi^{5} \mathrm{~d} \xi, \\
& \frac{T}{2}=\frac{\alpha \mathrm{e}^{\mathrm{i} \lambda K l}-\beta}{\lambda(\lambda+1)}+\frac{A_{1} \mathrm{e}^{\mathrm{i} \lambda_{1} K l}-A_{2}}{\lambda_{1}\left(\lambda_{1}+1\right)}+\frac{A_{3}-A_{4} \mathrm{e}^{-\mathrm{i} \bar{\lambda}_{1} K l}}{\bar{\lambda}_{1}\left(\bar{\lambda}_{1}+1\right)}+\frac{2 i}{\pi} \int_{0}^{\infty} \frac{B(\xi)-C(\xi) \mathrm{e}^{-\xi l}}{Q(\xi)} D K^{3} \xi{ }^{6} \mathrm{~d} \xi,
\end{aligned}
$$

where

$$
Q(\xi)=\left(\xi^{2}+K^{2}\right) P(\xi) .
$$

There are altogether eight equations in the above relations (2.12-2.19) involving 12 unknowns $A(\xi), B(\xi)$ etc. and the complete set of 12 equations for the determination of these unknowns are provided by the additional four equations, representing the four edge conditions, involving the two edges $x=0$ and $x=l$ which are given by:

$$
\begin{aligned}
& (\lambda K)^{3}\left(\alpha+\beta \mathrm{e}^{\mathrm{i} \lambda K l}\right)+\left(\lambda_{1} K\right)^{3}\left(A_{1}+A_{2} \mathrm{e}^{\mathrm{i} \lambda_{1} K l}\right)+\left(\bar{\lambda}_{1} K\right)^{3}\left(A_{3} \mathrm{e}^{-\mathrm{i} \bar{\lambda}_{1} K l}+A_{4}\right) \\
& -\frac{2 K}{\pi} \int_{0}^{\infty} \frac{B(\xi) \mathrm{e}^{-\xi l}+C(\xi)}{P(\xi)} \xi^{5} \mathrm{~d} \xi=0, \\
& (\lambda K)^{4}\left(\alpha-\beta \mathrm{e}^{\mathrm{i} \lambda K l}\right)+\left(\lambda_{1} K\right)^{4}\left(A_{1}-A_{2} \mathrm{e}^{\mathrm{i} \lambda_{1} K l}\right)+\left(\bar{\lambda}_{1} K\right)^{4}\left(A_{3} \mathrm{e}^{-\mathrm{i} \bar{\lambda}_{1} K l}-A_{4}\right) \\
& +\frac{2 K \mathrm{i}}{\pi} \int_{0}^{\infty} \frac{B(\xi) \mathrm{e}^{-\xi l}-C(\xi)}{P(\xi)} \xi^{6} \mathrm{~d} \xi=0, \\
& (\lambda K)^{3}\left(\alpha \mathrm{e}^{\mathrm{i} \lambda K l}+\beta\right)+\left(\lambda_{1} K\right)^{3}\left(A_{1} \mathrm{e}^{\mathrm{i} \lambda_{1} K l}+A_{2}\right)+\left(\bar{\lambda}_{1} K\right)^{3}\left(A_{3}+A_{4} \mathrm{e}^{-\mathrm{i} \bar{\lambda}_{1} K l}\right) \\
& -\frac{2 K}{\pi} \int_{0}^{\infty} \frac{B(\xi)+C(\xi) \mathrm{e}^{-\xi l}}{P(\xi)} \xi^{5} \mathrm{~d} \xi=0, \\
& (\lambda K)^{4}\left(\alpha \mathrm{e}^{\mathrm{i} \lambda K l}-\beta\right)+\left(\lambda_{1} K\right)^{4}\left(A_{1} \mathrm{e}^{\mathrm{i} \lambda_{1} K l}-A_{2}\right)+\left(\bar{\lambda}_{1} K\right)^{4}\left(A_{3}-A_{4} \mathrm{e}^{-\mathrm{i} \bar{\lambda}_{1} K l}\right) \\
& +\frac{2 K \mathrm{i}}{\pi} \int_{0}^{\infty} \frac{B(\xi)-C(\xi) \mathrm{e}^{-\xi l}}{P(\xi)} \xi^{6} \mathrm{~d} \xi=0 .
\end{aligned}
$$

Elimination of $A(\xi)$ between (2.12) and (2.13), and $G(\xi)$ between (2.14) and (2.15) gives rise to the following coupled Carleman-type singular integral equations for $B(\xi)$ and $C(\xi)$ :

$$
\mu(\xi) B_{1}(\xi)+\frac{1}{\pi} \int_{0}^{\infty} \frac{B_{1}(u)}{u-\xi} \mathrm{d} u-\frac{1}{\pi} \int_{0}^{\infty} \frac{C_{1}(u)}{u+\xi} \mathrm{e}^{-u l} \mathrm{~d} u=F_{B}(\xi), \quad \xi>0,
$$




$$
\mu(\xi) C_{1}(\xi)+\frac{1}{\pi} \int_{0}^{\infty} \frac{C_{1}(u)}{u-\xi} \mathrm{d} u-\frac{1}{\pi} \int_{0}^{\infty} \frac{B_{1}(u)}{u+\xi} \mathrm{e}^{-u l} \mathrm{~d} u=F_{C}(\xi), \quad \xi>0
$$

where

$$
\begin{aligned}
& \left(B_{1}(\xi), C_{1}(\xi)\right)=\frac{D K \xi^{5}}{P(\xi)}(B(\xi), C(\xi)), \\
& \mu(\xi)=\frac{\xi^{2}\left(D \xi^{4}+1\right)+K^{2}}{D K \xi^{5}}
\end{aligned}
$$

and

$$
\begin{aligned}
F_{B}(\xi)= & \frac{\lambda-1}{2 \lambda^{2} K}\left\{\frac{\alpha \mathrm{e}^{\mathrm{i} \lambda K l}}{\xi-\mathrm{i} K \lambda}+\frac{\beta}{\xi+\mathrm{i} K \lambda}\right\}+\frac{\lambda_{1}-1}{2 \lambda_{1}^{2} K}\left\{\frac{A_{1} \mathrm{e}^{\mathrm{i} \lambda_{1} K l}}{\xi-\mathrm{i} K \lambda_{1}}+\frac{A_{2}}{\xi+\mathrm{i} K \lambda_{1}}\right\} \\
& +\frac{\bar{\lambda}_{1}-1}{2 \bar{\lambda}_{1}^{2} K}\left\{\frac{A_{3}}{\xi-\mathrm{i} K \bar{\lambda}_{1}}+\frac{A_{4} \mathrm{e}^{-\mathrm{i} \bar{\lambda}_{1} K l}}{\xi+\mathrm{i} K \bar{\lambda}_{1}}\right\}, \\
F_{C}(\xi)= & \frac{\lambda-1}{2 \lambda^{2} K}\left\{\frac{\alpha}{\xi+\mathrm{i} K \lambda}+\frac{\beta \mathrm{e}^{\mathrm{i} \lambda K l}}{\xi-\mathrm{i} K \lambda}\right\}+\frac{\lambda_{1}-1}{2 \lambda_{1}^{2} K}\left\{\frac{A_{1}}{\xi+\mathrm{i} K \lambda_{1}}+\frac{A_{2} \mathrm{e}^{\mathrm{i} \lambda_{1} K l}}{\xi-\mathrm{i} K \lambda_{1}}\right\} \\
& +\frac{\bar{\lambda}_{1}-1}{2 \bar{\lambda}_{1}^{2} K}\left\{\frac{A_{3} \mathrm{e}^{-\mathrm{i} \bar{\lambda}_{1} K l}}{\xi+\mathrm{i} K \bar{\lambda}_{1}}+\frac{A_{4}}{\xi-\mathrm{i} K \bar{\lambda}_{1}}\right\} .
\end{aligned}
$$

We note that $F_{B}(\xi)$ and $F_{C}(\xi)$ involve the unknown constants $\alpha, \beta, A_{1}, A_{2}, A_{3}$ and $A_{4}$. In the next section we describe a method to obtain approximate solutions for $B_{1}(\xi), C_{1}(\xi)$.

\section{Approximate solutions}

In this section, we shall describe an approximate iterative method of solution of the system of coupled integral Equations (2.25) and (2.26), when $l$ is large. If we take the strip width $l$ to be so large that the terms which make the Equations (2.25) and (2.26) coupled vanish, then we get two uncoupled equations for $B_{1}^{0}(\xi)$ and $C_{1}^{0}(\xi)$, as given by

$$
\begin{aligned}
& \mu(\xi) B_{1}^{0}(\xi)+\frac{1}{\pi} \int_{0}^{\infty} \frac{B_{1}^{0}(u)}{u-\xi} \mathrm{d} u=F_{B}^{0}(\xi), \quad \xi>0, \\
& \mu(\xi) C_{1}^{0}(\xi)+\frac{1}{\pi} \int_{0}^{\infty} \frac{C_{1}^{0}(u)}{u-\xi} \mathrm{d} u=F_{C}^{0}(\xi), \quad \xi>0,
\end{aligned}
$$

for the zero-order approximations $B_{1}^{0}$ and $C_{1}^{0}$ of the functions $B_{1}$ and $C_{1}$, where $F_{B}^{0}(\xi)$ and $F_{C}^{0}(\xi)$ are given by (2.28) and (2.29) with the unknown constants $\alpha, \beta$ etc. replaced by $\alpha^{0}, \beta^{0}$ etc. and neglecting the terms involving $\mathrm{e}^{\mathrm{i} \lambda_{1} K l}$ and $\mathrm{e}^{-\mathrm{i} \bar{\lambda}_{1} K l}$ as these are then exponentially small for large $l$. Now we can solve the integral equations (3.1) and (3.2) by converting them into Riemann-Hilbert problems (see [14]). We find that the solutions of the integral Equations (3.1) and (3.2) are given by:

$$
B_{1}^{0}(\xi)=\frac{\lambda-1}{2 \lambda^{2} K}\left\{\alpha^{0} \mathrm{e}^{\mathrm{i} \lambda K l} P_{\alpha}(\xi)+\beta^{0} P_{\beta}(\xi)\right\}+\frac{\lambda_{1}-1}{2 \lambda_{1}^{2} K} A_{2}^{0} P_{2}(\xi)+\frac{\bar{\lambda}_{1}-1}{2 \bar{\lambda}_{1}^{2} K} A_{3}^{0} P_{3}(\xi)
$$


and

$$
C_{1}^{0}(\xi)=\frac{\lambda-1}{2 \lambda^{2} K}\left\{\alpha^{0} P_{\beta}(\xi)+\beta^{0} \mathrm{e}^{\mathrm{i} \lambda K l} P_{\alpha}(\xi)\right\}+\frac{\lambda_{1}-1}{2 \lambda_{1}^{2} K} A_{1}^{0} P_{2}(\xi)+\frac{\bar{\lambda}_{1}-1}{2 \bar{\lambda}_{1}^{2} K} A_{4}^{0} P_{3}(\xi)
$$

where $P_{\alpha}(\xi)$ is given by

$$
P_{\alpha}(\xi)=\frac{\Lambda_{0}^{+}(\xi)}{\mu(\xi)-\mathrm{i}}\left\{\frac{\mu(\xi)}{\Lambda_{0}^{+}(\xi)(\mu(\xi)+\mathrm{i})} \frac{1}{\xi-\mathrm{i} K \lambda}-\frac{1}{\pi} \int_{0}^{\infty} \frac{\mathrm{d} u}{\Lambda_{0}^{+}(u)(\mu(u)+\mathrm{i})(u-\xi)(u-\mathrm{i} K \lambda)}\right\}
$$

with similar expressions representing the other $P$-functions.

Also

$$
\Lambda_{0}(\zeta)=\exp \left[\frac{1}{2 \pi \mathrm{i}} \int_{0}^{\infty}\left\{\left(\log \left[\frac{\mu(t)-\mathrm{i}}{\mu(t)+\mathrm{i}}\right]-\lim _{\mathrm{t} \rightarrow \infty} \log \left[\frac{\mu(t)-\mathrm{i}}{\mu(t)+\mathrm{i}}\right]\right) /(t-\zeta)\right\} \mathrm{d} t\right], \quad \zeta \notin(0, \infty)
$$

holds good, along with

$$
\Lambda^{ \pm}(\xi)= \pm \frac{1}{2} B_{1}^{0}(\xi)+\frac{1}{2 \pi \mathrm{i}} \int_{0}^{\infty} \frac{B_{1}^{0}(u)}{u-\xi} \mathrm{d} u, \quad \xi>0 .
$$

Now, by considering the integral

$$
\int_{\Gamma_{0}} \frac{1}{\Lambda_{0}(\tau)(\tau-\mathrm{i} K \lambda)} \frac{\mathrm{d} \tau}{\tau-\zeta}, \quad \zeta \notin \Gamma_{0},
$$

where $\Gamma_{0}$ is a positively oriented contour consisting of a loop around the positive real axis having indentations above the point $\tau=\xi+\mathrm{i} 0$ and below the point $\tau=\xi-\mathrm{i} 0$ and a circle of large radius with centre at the origin, in the complex $\tau$-plane, we can evaluate the integral in the expression for $P_{\alpha}(\xi)$. Thus we obtain

$$
P_{\alpha}(\xi)=\frac{\Lambda_{0}^{+}(\xi)}{\mu(\xi)-\mathrm{i}} \frac{1}{\Lambda_{0}(\mathrm{i} K \lambda)(\xi-\mathrm{i} K \lambda)} .
$$

$P_{\beta}(\xi), P_{2}(\xi)$ and $P_{3}(\xi)$ are obtained in a similar manner.

To determine the zero-order approximations to the constants, we use Equations (2.16-2.19) and (2.21-2.24). By using the relations (2.27a) in these equations, and then making $l$ large enough, so that $B_{1}(\xi)$ and $C_{1}(\xi)$ can be replaced by $B_{1}^{0}(\xi)$ and $C_{1}^{0}(\xi)$, respectively, we obtain the following eight equations for the determination of the eight unknowns $R^{0}, T^{0}, \alpha^{0}$ etc.:

$$
\begin{aligned}
& \frac{1+R^{0}}{2}=\frac{\alpha^{0}+\beta^{0} \mathrm{e}^{\mathrm{i} \lambda K l}}{\lambda^{2}(\lambda+1)}+\frac{A_{1}^{0}}{\lambda_{1}^{2}\left(\lambda_{1}+1\right)}+\frac{A_{4}^{0}}{\bar{\lambda}_{1}^{2}\left(\bar{\lambda}_{1}+1\right)}-\frac{2 K^{3}}{\pi} \int_{0}^{\infty} \frac{C_{1}^{0}(\xi)}{\xi^{2}+K^{2}} \mathrm{~d} \xi, \\
& \frac{1-R^{0}}{2}=\frac{\alpha^{0}-\beta^{0} \mathrm{e}^{\mathrm{i} \lambda K l}}{\lambda(\lambda+1)}+\frac{A_{1}^{0}}{\lambda_{1}\left(\lambda_{1}+1\right)}-\frac{A_{4}^{0}}{\bar{\lambda}_{1}\left(\bar{\lambda}_{1}+1\right)}-\frac{2 K^{2} \mathrm{i}}{\pi} \int_{0}^{\infty} \frac{\xi C_{1}^{0}(\xi)}{\xi^{2}+K^{2}} \mathrm{~d} \xi, \\
& \frac{T^{0}}{2}=\frac{\alpha^{0} \mathrm{e}^{\mathrm{i} \lambda K l}+\beta^{0}}{\lambda^{2}(\lambda+1)}+\frac{A_{2}^{0}}{\lambda_{1}^{2}\left(\lambda_{1}+1\right)}+\frac{A_{3}^{0}}{\bar{\lambda}_{1}^{2}\left(\bar{\lambda}_{1}+1\right)}-\frac{2 K^{3}}{\pi} \int_{0}^{\infty} \frac{B_{1}^{0}(\xi)}{\xi^{2}+K^{2}} \mathrm{~d} \xi, \\
& \frac{T^{0}}{2}=\frac{\alpha^{0} \mathrm{e}^{\mathrm{i} \lambda K l}-\beta^{0}}{\lambda(\lambda+1)}-\frac{A_{2}^{0}}{\lambda_{1}\left(\lambda_{1}+1\right)}+\frac{A_{3}^{0}}{\bar{\lambda}_{1}\left(\bar{\lambda}_{1}+1\right)}+\frac{2 K^{2} \mathrm{i}}{\pi} \int_{0}^{\infty} \frac{\xi B_{1}^{0}(\xi)}{\xi^{2}+K^{2}} \mathrm{~d} \xi,
\end{aligned}
$$




$$
\begin{aligned}
& (\lambda K)^{3}\left(\alpha^{0}+\beta^{0} \mathrm{e}^{\mathrm{i} \lambda K l}\right)+\left(\lambda_{1} K\right)^{3} A_{1}^{0}+\left(\bar{\lambda}_{1} K\right)^{3} A_{4}^{0}-\frac{2}{D \pi} \int_{0}^{\infty} C_{1}^{0}(\xi) \mathrm{d} \xi=0, \\
& (\lambda K)^{4}\left(\alpha^{0}-\beta^{0} \mathrm{e}^{\mathrm{i} \lambda K l}\right)+\left(\lambda_{1} K\right)^{4} A_{1}^{0}-\left(\bar{\lambda}_{1} K\right)^{4} A_{4}^{0}-\frac{2 i}{D \pi} \int_{0}^{\infty} \xi C_{1}^{0}(\xi) \mathrm{d} \xi=0, \\
& (\lambda K)^{3}\left(\alpha^{0} \mathrm{e}^{\mathrm{i} \lambda K l}+\beta^{0}\right)+\left(\lambda_{1} K\right)^{3} A_{2}^{0}+\left(\bar{\lambda}_{1} K\right)^{3} A_{3}^{0}-\frac{2}{D \pi} \int_{0}^{\infty} B_{1}^{0}(\xi) \mathrm{d} \xi=0, \\
& (\lambda K)^{4}\left(\alpha^{0} \mathrm{e}^{\mathrm{i} \lambda K l}-\beta^{0}\right)-\left(\lambda_{1} K\right)^{4} A_{2}^{0}+\left(\bar{\lambda}_{1} K\right)^{4} A_{3}^{0}+\frac{2 \mathrm{i}}{D \pi} \int_{0}^{\infty} \xi B_{1}^{0}(\xi) \mathrm{d} \xi=0 .
\end{aligned}
$$

Substitution of $B_{1}^{0}(\xi)$ and $C_{1}^{0}(\xi)$ from (3.3) and (3.4) in (3.9-3.16) will produce the following linear system of eight equations:

$$
\mathcal{A}^{0} X^{0}=\mathcal{B}
$$

where $\mathcal{A}^{0}=\left(a_{i j}^{0}\right)$ is an $8 \times 8$ matrix, $X^{0}$ and $\mathcal{B}$ are column vectors having the forms

$$
X^{0}=\left[\alpha^{0}, \beta^{0}, A_{1}^{0}, A_{2}^{0}, A_{3}^{0}, A_{4}^{0}, R^{0}, T^{0}\right]^{T}
$$

and

$$
\mathcal{B}=[1,1,0,0,0,0,0,0]^{T} .
$$

The matrix elements $a_{i j}^{0}$ 's are explicitly given in Appendix B. These elements will involve integrals of the form

$$
\int_{0}^{\infty} \frac{\Lambda_{0}^{+}(\xi)}{\mu(\xi)-\mathrm{i}} \frac{L_{1}(\xi)}{L_{2}(\xi)} \mathrm{d} \xi
$$

where $L_{1}(\xi)$ and $L_{2}(\xi)$ are polynomial expressions in $\xi$. These integrals are calculated by considering integrals of the following type

$$
\int_{\Gamma} \Lambda_{0}(\zeta) \frac{L_{1}(\zeta)}{L_{2}(\zeta)} \mathrm{d} \zeta
$$

where $\Gamma$ is a positively oriented closed contour consisting of a loop around the positive real axis and a circle of large radius, with centre at the origin, in the complex $\zeta$ plane. If the polynomials $L_{1}(\zeta), L_{2}(\zeta)$ are such that the contribution to the integral in (3.20) over the circle of large radius vanishes, then the integral in (3.20) becomes equal to

$-\pi$ (sum of residues of $\Lambda_{0} \frac{L_{1}}{L_{2}}$ at the poles lying inside $\Gamma$ ).

Since we can calculate the values of $\Lambda_{0}$ at different points by using the relation (3.5), the elements of the matrix $\mathcal{A}^{0}$ are completely determined. Thus, the zero-order approximations to the different constants are obtained by solving the matrix equation (3.17). Thus, the functions $B_{1}^{0}(\xi)$ and $C_{1}^{0}(\xi)$ are fully known from Equations (3.3) and (3.4), respectively.

To obtain the next higher-order (first-order) approximate solutions $B_{1}^{1}(\xi)$ and $C_{1}^{1}(\xi)$ of Equations (2.25) and (2.26), we substitute $C_{1}^{0}$ in place of $C_{1}$ in Equation (2.25) and $B_{1}^{0}$ in place of $B_{1}$ in Equation (2.26), giving the Carleman equations

$$
\mu(\xi) B_{1}^{1}(\xi)+\frac{1}{\pi} \int_{0}^{\infty} \frac{B_{1}^{1}(u)}{u-\xi} \mathrm{d} u=F_{B}^{1}(\xi), \quad \xi>0,
$$


and

$$
\mu(\xi) C_{1}^{1}(\xi)+\frac{1}{\pi} \int_{0}^{\infty} \frac{C_{1}^{1}(u)}{u-\xi} \mathrm{d} u=F_{C}^{1}(\xi), \quad \xi>0,
$$

where

$$
\begin{aligned}
F_{B}^{1}(\xi)= & \frac{1}{\pi} \int_{0}^{\infty} \frac{C_{1}^{0}(u) \mathrm{e}^{-u l}}{u+\xi} \mathrm{d} u+\frac{\lambda-1}{2 \lambda^{2} K}\left\{\frac{\alpha^{1} \mathrm{e}^{\mathrm{i} \lambda K l}}{\xi-\mathrm{i} K \lambda}+\frac{\beta^{1}}{\xi+\mathrm{i} K \lambda}\right\} \\
& +\frac{\lambda_{1}-1}{2 \lambda_{1}^{2} K}\left\{\frac{A_{1}^{1} \mathrm{e}^{\mathrm{i} \lambda_{1} K l}}{\xi-\mathrm{i} K \lambda_{1}}+\frac{A_{2}^{1}}{\xi+\mathrm{i} K \lambda_{1}}\right\}+\frac{\bar{\lambda}_{1}-1}{2 \bar{\lambda}_{1}^{2} K}\left\{\frac{A_{3}^{1}}{\xi-\mathrm{i} K \bar{\lambda}_{1}}+\frac{A_{4}^{1} \mathrm{e}^{-\mathrm{i} \bar{\lambda}_{1} K l}}{\xi+\mathrm{i} K \bar{\lambda}_{1}}\right\},
\end{aligned}
$$

and

$$
\begin{aligned}
F_{C}^{1}(\xi)= & \frac{1}{\pi} \int_{0}^{\infty} \frac{B_{1}^{0}(u) \mathrm{e}^{-u l}}{u+\xi} \mathrm{d} u+\frac{\lambda-1}{2 \lambda^{2} K}\left\{\frac{\alpha^{1}}{\xi+\mathrm{i} K \lambda}+\frac{\beta^{1} \mathrm{e}^{\mathrm{i} \lambda K l}}{\xi-\mathrm{i} K \lambda}\right\} \\
& +\frac{\lambda_{1}-1}{2 \lambda_{1}^{2} K}\left\{\frac{A_{1}^{1}}{\xi+\mathrm{i} K \lambda_{1}}+\frac{A_{2}^{1} \mathrm{e}^{\mathrm{i} \lambda_{1} K l}}{\xi-\mathrm{i} K \lambda_{1}}\right\}+\frac{\bar{\lambda}_{1}-1}{2 \bar{\lambda}_{1}^{2} K}\left\{\frac{A_{3}^{1} \mathrm{e}^{-\mathrm{i} \bar{\lambda}_{1} K l}}{\xi+\mathrm{i} K \bar{\lambda}_{1}}+\frac{A_{4}^{1}}{\xi-\mathrm{i} K \bar{\lambda}_{1}}\right\} .
\end{aligned}
$$

The integral equations (3.21) and (3.22) can be solved by using a technique similar to the one used to solve the Equations (3.1) and (3.2), and we find:

$$
\begin{aligned}
B_{1}^{1}(\xi)= & \frac{\lambda-1}{2 \lambda^{2} K}\left\{\alpha^{1} \mathrm{e}^{\mathrm{i} \lambda K l} P_{\alpha}(\xi)+\beta^{1} P_{\beta}(\xi)\right\}+\frac{\lambda_{1}-1}{2 \lambda_{1}^{2} K}\left\{A_{1}^{1} \mathrm{e}^{\mathrm{i} \lambda_{1} K l} P_{1}(\xi)+A_{2}^{1} P_{2}(\xi)\right\} \\
& +\frac{\bar{\lambda}_{1}-1}{2 \bar{\lambda}_{1}^{2} K}\left\{A_{3}^{1} P_{3}(\xi)+A_{4}^{1} \mathrm{e}^{-\mathrm{i} \overline{\lambda_{1}} K l} P_{4}(\xi)\right\}
\end{aligned}
$$

and

$$
\begin{aligned}
C_{1}^{1}(\xi)= & \frac{\lambda-1}{2 \lambda^{2} K}\left\{\alpha^{1} P_{\beta}(\xi)+\beta^{1} \mathrm{e}^{\mathrm{i} \lambda K l} P_{\alpha}(\xi)\right\}+\frac{\lambda_{1}-1}{2 \lambda_{1}^{2} K}\left\{A_{1}^{1} P_{2}(\xi)+A_{2}^{1} \mathrm{e}^{\mathrm{i} \lambda_{1} K l} P_{1}(\xi)\right\} \\
& +\frac{\bar{\lambda}_{1}-1}{2 \bar{\lambda}_{1}^{2} K}\left\{A_{3}^{1} \mathrm{e}^{-\mathrm{i} \bar{\lambda}_{1} K l} P_{4}(\xi)+A_{4}^{1} P_{3}(\xi)\right\}
\end{aligned}
$$

where the superscript ' 1 ' denotes the first-order approximation to the corresponding quantities. It may be noted that, to arrive at the results (3.25) and (3.26), we utilized results of integrals of the form

$$
\int_{\Gamma_{0}} \frac{g(\tau), h(\tau)}{\Lambda_{0}(\tau)(\tau-\zeta)} \mathrm{d} \tau, \quad \zeta \notin \Gamma_{0},
$$

where

$$
(g, h)(\tau)=\frac{2}{\pi} \int_{0}^{\infty} \frac{\left(B_{1}^{0}, C_{1}^{0}\right)(u) \mathrm{e}^{-u l}}{u+\tau} \mathrm{d} u,
$$

which can be taken to be zero approximately for large $l$. This means that there is no contribution to $B_{1}^{1}(\xi)$ and $C_{1}^{1}(\xi)$ arising from the integrals in (3.23) and (3.24).

Since $B_{1}^{1}(\xi)$ and $C_{1}^{1}(\xi)$ are now obtained in terms of the unknown constants $\alpha^{1}, \beta^{1}, A_{1}^{1}, A_{2}^{1}$, $A_{3}^{1}, A_{4}^{1}$, use of (2.27a) in the Equations (2.16-2.19) and (2.21-2.24) produces the following 
eight equations for the first-order approximations to the the eight unknown constants, viz. $\alpha^{1}, \beta^{1}, A_{1}^{1}, A_{2}^{1}, A_{3}^{1}, A_{4}^{1}, R^{1}$ and $T^{1}$ :

$$
\begin{aligned}
& \frac{1+R^{1}}{2}=\frac{\alpha^{1}+\beta^{1} \mathrm{e}^{\mathrm{i} \lambda K l}}{\lambda^{2}(\lambda+1)}+\frac{A_{1}^{1}+A_{2}^{1} \mathrm{e}^{\mathrm{i} \lambda_{1} K l}}{\lambda_{1}^{2}\left(\lambda_{1}+1\right)}+\frac{A_{3}^{1} \mathrm{e}^{-\mathrm{i} \bar{\lambda}_{1} K l}+A_{4}^{1}}{\bar{\lambda}_{1}^{2}\left(\bar{\lambda}_{1}+1\right)} \\
& -\frac{2 K^{3}}{\pi} \int_{0}^{\infty} \frac{B_{1}^{1}(\xi) \mathrm{e}^{-\xi l}+C_{1}^{1}(\xi)}{\xi^{2}+K^{2}} \mathrm{~d} \xi \\
& \frac{1-R^{1}}{2}=\frac{\alpha^{1}-\beta^{1} \mathrm{e}^{\mathrm{i} \lambda K l}}{\lambda(\lambda+1)}+\frac{A_{1}^{1}-A_{2}^{1} \mathrm{e}^{\mathrm{i} \lambda_{1} K l}}{\lambda_{1}\left(\lambda_{1}+1\right)}+\frac{A_{3}^{1} \mathrm{e}^{-\mathrm{i} \bar{\lambda}_{1} K l}-A_{4}^{1}}{\bar{\lambda}_{1}\left(\bar{\lambda}_{1}+1\right)} \\
& +\frac{2 K^{2} \mathrm{i}}{\pi} \int_{0}^{\infty} \frac{B_{1}^{1}(\xi) \mathrm{e}^{-\xi l}-C_{1}^{1}(\xi)}{\xi^{2}+K^{2}} \xi \mathrm{d} \xi, \\
& \frac{T^{1}}{2}=\frac{\alpha^{1} \mathrm{e}^{\mathrm{i} \lambda K l}+\beta^{1}}{\lambda^{2}(\lambda+1)}+\frac{A_{1}^{1} \mathrm{e}^{\mathrm{i} \lambda_{1} K l}+A_{2}^{1}}{\lambda_{1}^{2}\left(\lambda_{1}+1\right)}+\frac{A_{3}^{1}+A_{4}^{1} \mathrm{e}^{-\mathrm{i} \bar{\lambda}_{1} K l}}{\bar{\lambda}_{1}^{2}\left(\bar{\lambda}_{1}+1\right)} \\
& -\frac{2 K^{3}}{\pi} \int_{0}^{\infty} \frac{B_{1}^{1}(\xi)+C_{1}^{1}(\xi) \mathrm{e}^{-\xi l}}{\xi^{2}+K^{2}} \mathrm{~d} \xi, \\
& \frac{T^{1}}{2}=\frac{\alpha^{1} \mathrm{e}^{\mathrm{i} \lambda K l}-\beta^{1}}{\lambda(\lambda+1)}+\frac{A_{1}^{1} \mathrm{e}^{\mathrm{i} \lambda_{1} K l}-A_{2}^{1}}{\lambda_{1}\left(\lambda_{1}+1\right)}+\frac{A_{3}^{1}-A_{4}^{1} \mathrm{e}^{-\mathrm{i} \bar{\lambda}_{1} K l}}{\bar{\lambda}_{1}\left(\bar{\lambda}_{1}+1\right)} \\
& +\frac{2 K^{2} \mathrm{i}}{\pi} \int_{0}^{\infty} \frac{B_{1}^{1}(\xi)-C_{1}^{1}(\xi) \mathrm{e}^{-\xi l}}{\xi^{2}+K^{2}} \xi \mathrm{d} \xi, \\
& (\lambda K)^{3}\left(\alpha^{1}+\beta^{1} \mathrm{e}^{\mathrm{i} \lambda K l}\right)+\left(\lambda_{1} K\right)^{3}\left(A_{1}^{1}+A_{2}^{1} \mathrm{e}^{\mathrm{i} \lambda_{1} K l}\right)+\left(\bar{\lambda}_{1} K\right)^{3}\left(A_{3}^{1} \mathrm{e}^{-\mathrm{i} \bar{\lambda}_{1} K l}+A_{4}^{1}\right) \\
& -\frac{2}{D \pi} \int_{0}^{\infty}\left\{B_{1}^{1}(\xi) \mathrm{e}^{-\xi l}+C_{1}^{1}(\xi)\right\} \mathrm{d} \xi=0, \\
& (\lambda K)^{4}\left(\alpha^{1}-\beta^{1} \mathrm{e}^{\mathrm{i} \lambda K l}\right)+\left(\lambda_{1} K\right)^{4}\left(A_{1}^{1}-A_{2}^{1} \mathrm{e}^{\mathrm{i} \lambda_{1} K l}\right)+\left(\bar{\lambda}_{1} K\right)^{4}\left(A_{3}^{1} \mathrm{e}^{-\mathrm{i} \bar{\lambda}_{1} K l}-A_{4}^{1}\right) \\
& +\frac{2 i}{D \pi} \int_{0}^{\infty}\left\{B_{1}^{1}(\xi) \mathrm{e}^{-\xi l}-C_{1}^{1}(\xi)\right\} \xi \mathrm{d} \xi=0, \\
& (\lambda K)^{3}\left(\alpha^{1} \mathrm{e}^{\mathrm{i} \lambda K l}+\beta^{1}\right)+\left(\lambda_{1} K\right)^{3}\left(A_{1}^{1} \mathrm{e}^{\mathrm{i} \lambda_{1} K l}+A_{2}^{1}\right)+\left(\bar{\lambda}_{1} K\right)^{3}\left(A_{3}^{1}+A_{4}^{1} \mathrm{e}^{-\mathrm{i} \bar{\lambda}_{1} K l}\right) \\
& -\frac{2}{D \pi} \int_{0}^{\infty}\left\{B_{1}^{1}(\xi)+C_{1}^{1}(\xi) \mathrm{e}^{-\xi l}\right\} \mathrm{d} \xi=0 \\
& (\lambda K)^{4}\left(\alpha^{1} \mathrm{e}^{\mathrm{i} \lambda K l}-\beta^{1}\right)+\left(\lambda_{1} K\right)^{4}\left(A_{1}^{1} \mathrm{e}^{\mathrm{i} \lambda \lambda_{1} K l}-A_{2}^{1}\right)+\left(\bar{\lambda}_{1} K\right)^{4}\left(A_{3}^{1}-A_{4}^{1} \mathrm{e}^{-\mathrm{i} \bar{\lambda}_{1} K l}\right) \\
& +\frac{2 \mathrm{i}}{D \pi} \int_{0}^{\infty}\left\{B_{1}^{1}(\xi)-C_{1}^{1}(\xi) \mathrm{e}^{-\xi l}\right\} \xi \mathrm{d} \xi=0 .
\end{aligned}
$$

In order to calculate the coefficients in the above equations, we have to calculate integrals of the type (3.20). In addition we have to evaluate, approximately for large $l$, the following types of integrals:

$$
\int_{0}^{\infty} \frac{\Lambda_{0}^{+}(\xi)}{\mu(\xi)-\mathrm{i}} \frac{L_{1}(\xi)}{L_{2}(\xi)} \mathrm{e}^{-\xi l} \mathrm{~d} \xi .
$$


This is achieved by using the Watson's lemma $(c f .[16$, p. 438]). For this we require factorization of the function $[\mu(\xi)-\mathrm{i}]$ as

$$
\begin{aligned}
\mu(\xi)-\mathrm{i}= & \frac{1}{D K \xi^{5}}(\xi-\mathrm{i} K)(\xi+\mathrm{i} K \lambda)\left(\xi+\mathrm{i} K \lambda_{1}\right)\left(\xi+\mathrm{i} K \bar{\lambda}_{1}\right) \\
& \times\left(\xi+\mathrm{i} K \lambda_{2}\right)\left(\xi+\mathrm{i} K \bar{\lambda}_{2}\right) .
\end{aligned}
$$

The results will involve $\Lambda_{0}( \pm \mathrm{i} K), \Lambda_{0}( \pm \mathrm{i} K \lambda), \Lambda_{0}\left( \pm \mathrm{i} K \lambda_{1}\right), \Lambda_{0}\left( \pm \mathrm{i} K \bar{\lambda}_{1}\right), \Lambda_{0}^{+}(0)$ and $\Lambda_{0}^{+\prime}(0)$ (prime denotes differentiation) which are calculated from the expression (3.5) of $\Lambda_{0}(\zeta)$. Thus the firstorder corrections to the unknown constants are obtained. Utilizing these in Equations (3.25) and (3.26), we finally obtain $B_{1}^{1}(\xi)$ and $C_{1}^{1}(\xi)$ completely. Then from Equation (2.12) or (2.13) and from Equation (2.14) or (2.15) the first-order approximations to the functions $A(\xi)$ and $G(\xi)$ are obtained. The second- and other higher-order approximations to the unknown constants and functions can be obtained by repeating this iterative process. The numerical study based on the first-order approximate values $R^{1}$ and $T^{1}$ of $R$ and $T$ shows that the identity $\left|R^{1}\right|^{2}+\left|T^{1}\right|^{2}=1$ is almost true for various values of the different parameters of the problem. Thus, the iterative procedure explained above has produced an approximate solution of the problem under consideration, with the use of the first-order approximations and hence further higher-order approximations have not been carried out here.

\section{Numerical results}

In this section the graphs of $\left|R^{0}\right|,\left|R^{1}\right|,\left|T^{0}\right|$ and $\left|T^{1}\right|$ are analyzed in order to understand the difference between the zero-order and the first-order approximate values of the reflection and transmission coefficients. For numerical computations a characteristic length $L$ proportional to the wavelength is introduced in order to make the different parameters non-dimensional. Thus, $K L, l / L$ and $D / L^{4}$ are, respectively, the dimensionless wave number, strip width and ice-cover parameter.

In the Figures 1 and $2,\left|R^{0}\right|$ and $\left|R^{1}\right|$ are compared for two different width parameters $l / L=10,100$ against the wave number $K L$; the ice-cover parameter being kept fixed at the very small value $D / L^{4}=0.001$. From Figure 1 it may be observed that the curve of $\left|R^{1}\right|$ always lies above the curve of $\left|R^{0}\right|$. However, as the strip width increases, Figure 2 shows that the differences between the values of $\left|R^{0}\right|$ and $\left|R^{1}\right|$ decrease. Again, these two figures indicate that the reflection coefficient is small enough when the ice-cover parameter is very small, irrespective of the strip-width. As $D / L^{4}$ decreases, the effect of the ice-strip on the incoming waves becomes weaker. As a result, a major part of the incident wave is transmitted through the edges of the ice-sheet.

The graphs of $\left|R^{1}\right|$ for different values of the ice-cover parameter $D / L^{4}$ and for $l / L=10$ are plotted in Figure 3. This figure indicates that $\left|R^{1}\right|$ gradually increases with the increase in $D / L^{4}$. If the elastic parameters $(E$ and $v)$ are assumed to be fixed, the only factor that affects $D$ is the thickness of the ice-sheet. Thus, from this figure we may draw the conclusion that, if ice-sheets composed of homogeneous material (in the sense that $E$ and $v$ are constants throughout the material) are considered, those with a greater thickness, i.e., heavier ice-strips, exert more resistance on the incident wave.

Figures 4 and 5 display $\left|R^{0}\right|,\left|R^{1}\right|,\left|T^{0}\right|$ and $\left|T^{1}\right|$ against the ice-cover parameter $D / L^{4}$ for $l / L=10$, and for two different wavenumbers $K L=1$ and $K L=2$, respectively. It may be noticed from these two figures that the distinction between the zero-order and the first-order 


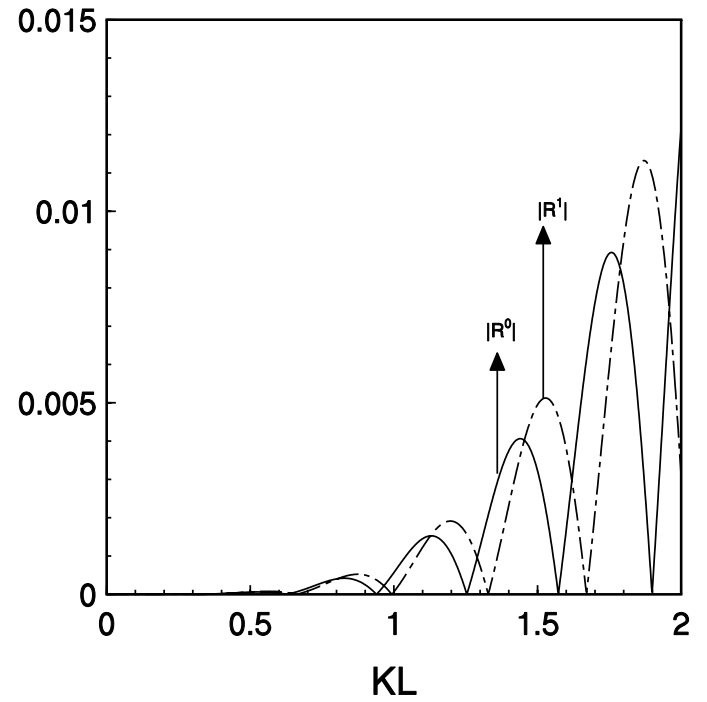

Figure 1. $\left|R^{0}\right|$ and $\left|R^{1}\right|$ for $1 / L=10$ and $D / L^{4}=$ 0.001 .

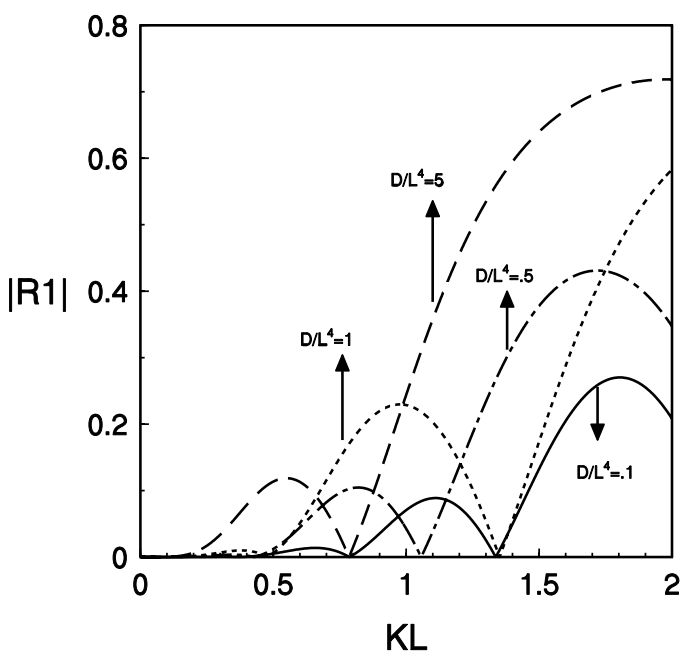

Figure 3. $\left|R^{1}\right|$ for different values of $D / L^{4}, I / L=10$.

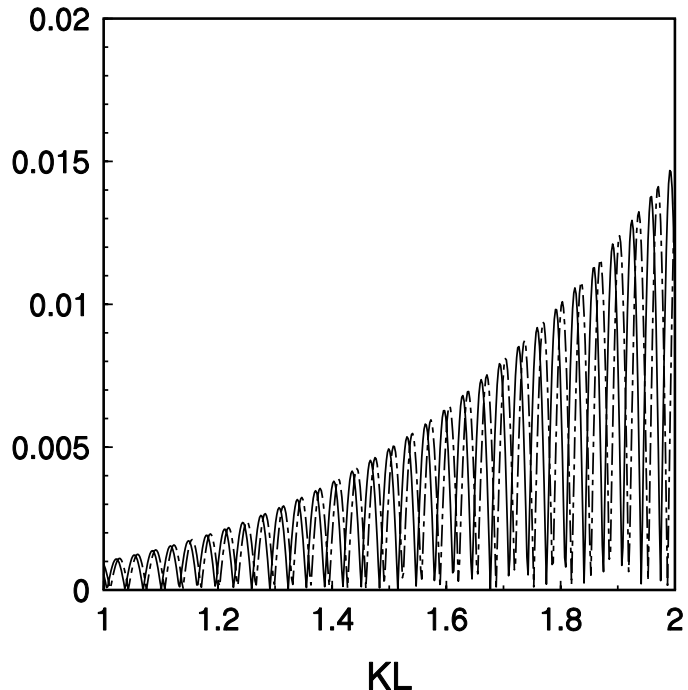

Figure 2. $\left|R^{0}\right|(--)$ and $\left|R^{1}\right|(--)$ for $1 / L=100$ and $D / L^{4}=0.001$.

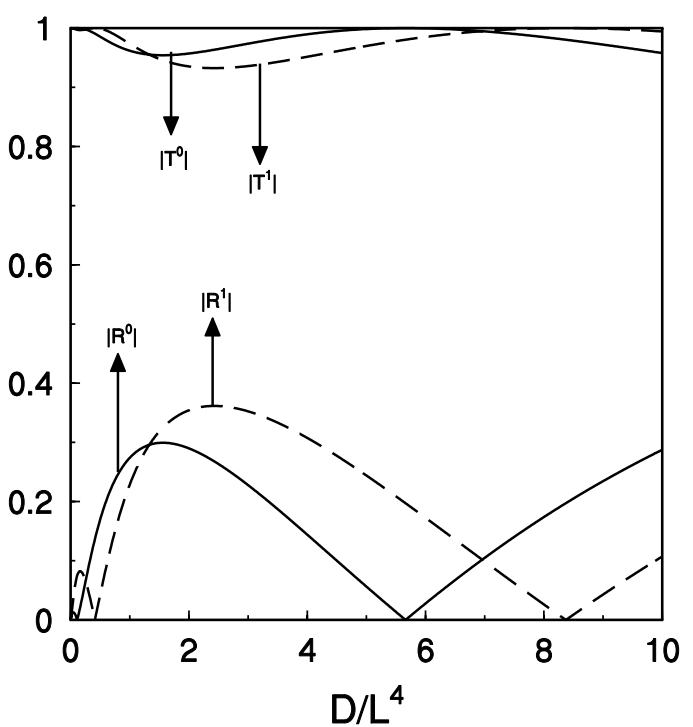

Figure 4. $\left|R^{0}\right|,\left|R^{1}\right|,\left|T^{0}\right|$ and $\left|T^{1}\right|$ for $I / L=10$ and $K L=1$.

approximations to the physical quantities $(R$ and $T)$ diminishes for larger wavenumber. Also, when $K L=2$, the reflection coefficient is larger than that for $K L=1$. The above two features are seen to hold good, even when the width parameter $l / L$ increases to 100 . This has been depicted in Figures 6 and 7. However, the number of zeros of the reflection coefficient increases for larger strip width. Thus, multiple reflections by the two edges of the ice-sheet increase with the increase in its width. Note that the phenomenon of occurrence of more multiple reflections for a wider strip was also seen to hold when $\left|R^{0}\right|$ and $\left|R^{1}\right|$ were depicted against the wavenumber for small $D / L^{4}$ (Figures 1 and 2). 


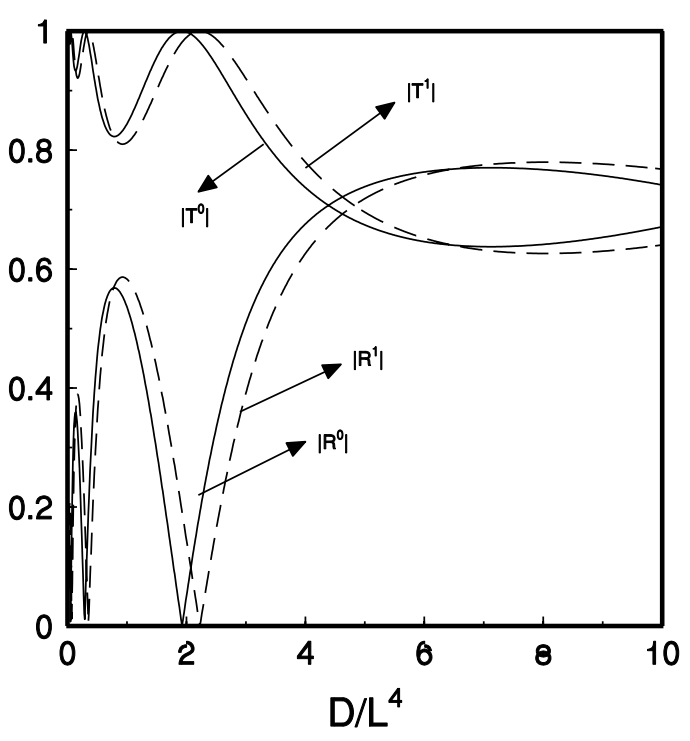

Figure 5. $\left|R^{0}\right|, \quad\left|R^{1}\right|, \quad\left|T^{0}\right|, \quad\left|T^{1}\right|$ for $I / L=10$ and $K L=2$.

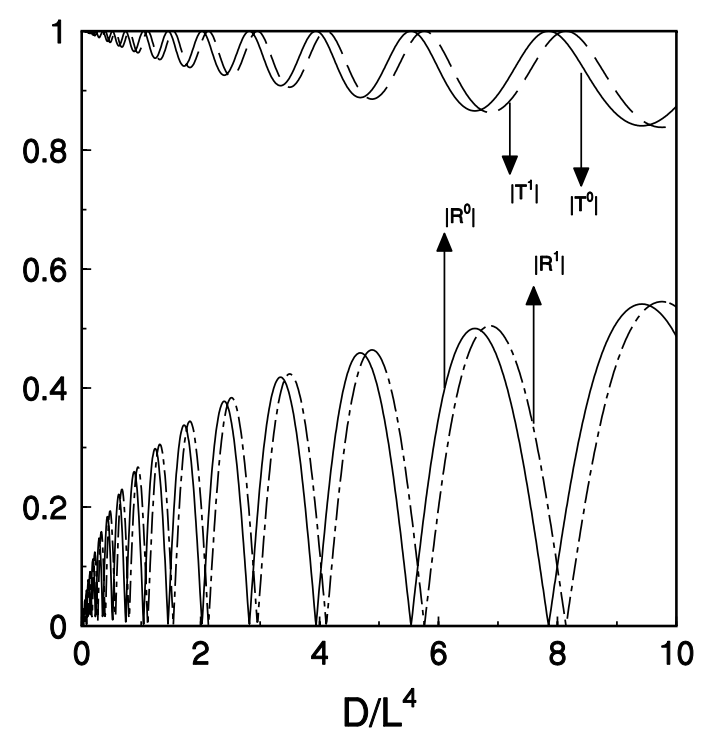

Figure 6. $\left|R^{0}\right|,\left|R^{1}\right|,\left|T^{0}\right|,\left|T^{1}\right|$ for $I / L=100$ and $K L=1$.

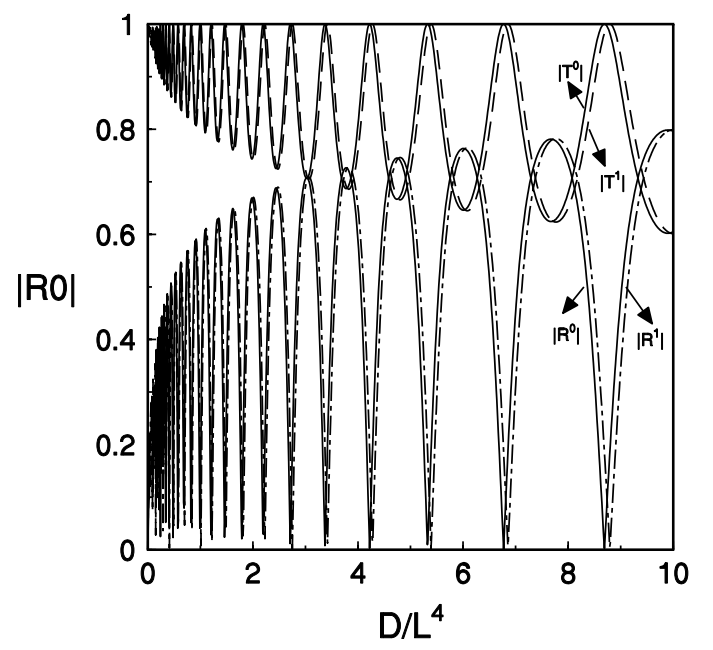

Figure 7. $\left|R^{0}\right|,\left|R^{1}\right|,\left|T^{0}\right|$ and $\left|T^{1}\right|$ for $I / L=100$ and $K L=2$.

\section{Conclusion}

The boundary-value problem describing the scattering of surface water waves by a strip of ice-cover of finite width has been considered for a suitable mathematical treatment, for its complete solution. The problem is reduced to two coupled singular integral equations of the Carleman type which were solved approximately for large strip width. As is well known (cf. [16, Section 9.12]), the problems of scattering of electromagnetic and acoustic waves, by strips of finite width, can not be solved in closed form and an approximate method of solution has been devised by Jones [16] to solve such scattering problems which are valid for large values of the width of the strip, and in order to obtain acceptable solutions valid for any finite 
width, one has to carry out a large number of iterations producing such approximate solutions.

The Wiener-Hopf technique is believed to be the most powerful tool to attack the class of scattering problems, for which the basic partial differential equation happens to be the Helmholtz's equation. The problem of scattering by a strip is reduced to a threepart Wiener-Hopf problem that cannot be solved in closed form, like the standard two-part Wiener-Hopf problem. Hence, approximate methods of solution of such scattering problems are the only possibilities. In the case of problems of scattering of surface water waves one encounters Laplace's equation instead of Helmholtz's equation as the basic partial differential equation and, in order to employ the Wiener-Hopf technique to handle such boundaryvalue problems, a special limiting procedure $(c f .[17,18])$ was found to be useful. In a previous study (cf. [18]), the problem of scattering of surface water waves by a rigid strip was investigated and an approximate solution of the boundary-value problem was obtained by using a limiting process along with the Wiener-Hopf technique. The problem treated in the present work is a generalization of the previous one, in the sense that the rigid strip is replaced by a strip of ice cover, producing a more complicated boundary-value problem. A more natural method of employing a generalized Havelock-type expansion, along with the application of the techniques of solving systems of singular integral equations, involving a large parameter, by the aid of Riemann-Hilbert problems and related mathematical ideas, is employed to handle the present general problem in the theory of scattering of surface water waves, by floating ice-strips. The numerical results for the reflection and transmission coefficients have been determined for a number of choices of the basic parameters of the problem and these results have been presented graphically. As a check on our results, we have always verified the energy criterion $\left(|R|^{2}+|T|^{2}=1\right)$, ensuring the acceptability of the approximate method used. The major feature of the curves for the reflection coefficients is that these are oscillatory in nature, which can be attributed to multiple reflections due to the two edges of the ice-strip.

\section{Acknowledgement}

The authors thank the referees and the Editor-in-Chief for their comments and suggestions to revise the paper in the present form. This work is partially supported by CSIR, India.

\section{APPENDIX A}

In deriving the ice-cover condition (2.3), if the assumption that waves are long compared to the thickness is not made, we find that the ice-cover condition takes the form

$$
K \phi+\left(D \partial_{x}^{4}+1-\epsilon K\right) \phi_{y}=0 \quad \text { on } y=0,
$$

where $\epsilon \rho$ is the constant surface density of the ice-cover, $\rho$ is the density of water and $\epsilon$ is a constant having dimension of length. For $1-\epsilon K>0$, the condition (A1) can be modified as

$$
K^{*} \phi+\left(D^{*} \partial_{x}^{4}+1\right) \phi_{y}=0 \quad \text { on } y=0
$$

where $K^{*}=K /(1-\epsilon K), D^{*}=D /(1-\epsilon K)$. This is of the same form as (2.3). For $1-\epsilon K<0$, the condition (A1) can be rewritten as

$$
K^{\prime} \phi+\left(D^{\prime} \partial_{x}^{4}-1\right) \phi_{y}=0 \quad \text { on } y=0
$$


where

$$
K^{\prime}=K /(\epsilon K-1), \quad D^{\prime}=D /(\epsilon K-1) .
$$

For deep water, $\phi(x, y)$ has the wave type solution

$$
\phi(x, y)=\mathrm{e}^{-k y \pm \mathrm{i} k x} .
$$

Then, the wave parameter $k$ satisfies

$$
K-\left(D k^{4}+1-\epsilon K\right) k=0 .
$$

The above equation always possesses a real positive root whether $1-\epsilon K$ is positive or negative ( $c f$. [2]) so long as $D \neq 0$. Thus, without any loss of generality, the ice-cover condition can be taken in the form given by (2.3) for $1-\epsilon K>0$. For $1-\epsilon K<0$, the only change in (2.3) is that the plus sign before $\phi_{y}$ is to be replaced by a minus sign but, as the nature of the roots of (A4) remains the same, a similar analysis can be made with a slight modification.

\section{APPENDIX B}

The matrix $\mathcal{A}^{0}$ appearing in (3.17) has the form

$$
\left(\begin{array}{llllllrl}
a_{\beta} & a_{\alpha} \mathrm{e}^{\mathrm{i} \lambda K l} & a_{2} & 0 & 0 & a_{3} & -1 & 0 \\
b_{\beta} & -b_{\alpha} \mathrm{e}^{\mathrm{i} \lambda K l} & b_{2} & 0 & 0 & -b_{3} & 1 & 0 \\
a_{\alpha} \mathrm{e}^{\mathrm{i} \lambda K l} & a_{\beta} & 0 & a_{2} & a_{3} & 0 & 0 & -1 \\
b_{\alpha} \mathrm{e}^{\mathrm{i} \lambda K l} & -b_{\beta} & 0 & b_{2} & b_{3} & 0 & 0 & -1 \\
c_{\alpha} & c_{\alpha} \mathrm{e}^{\mathrm{i} \lambda K l} & c_{1} & 0 & 0 & c_{3} & 0 & 0 \\
\lambda K c_{\alpha} & -\lambda K c_{\alpha} \mathrm{e}^{\mathrm{i} \lambda K l} \lambda_{1} K c_{1} & 0 & 0 & -\lambda_{1} K c_{3} & 0 & 0 \\
c_{\alpha} \mathrm{e}^{\mathrm{i} \lambda K l} & c_{\alpha} & 0 & c_{1} & c_{3} & 0 & 0 & 0 \\
\lambda K c_{\alpha} \mathrm{e}^{\mathrm{i} \lambda K l}-\lambda K c_{\alpha} & 0 & -\lambda_{1} K c_{1} \lambda_{1}^{-} K c_{3} 0 & 0 & 0
\end{array}\right),
$$

where

$$
\begin{aligned}
& a_{\alpha, \beta}=\frac{2 K^{3}}{(\lambda K)^{2}(\lambda K+K)}-\frac{2 K^{3}(\lambda K-K)}{\pi(\lambda K)^{2}} I_{\alpha, \beta}, \quad a_{1,2}=\frac{2 K^{3}}{\left(\lambda_{1} K\right)^{2}\left(\lambda_{1} K+K\right)}-\frac{2 K^{3}\left(\lambda_{1} K-K\right)}{\pi\left(\lambda_{1} K\right)^{2}} I_{1,2}, \\
& a_{3,4}=\frac{2 K^{3}}{\left(\bar{\lambda}_{1} K\right)^{2}\left(\bar{\lambda}_{1} K+K\right)}-\frac{2 K^{3}\left(\bar{\lambda}_{1} K-K\right)}{\pi\left(\bar{\lambda}_{1} K\right)^{2}} I_{3,4}, \quad b_{\alpha, \beta}=\frac{2 K^{2}}{\lambda K(\lambda K+K)} \pm \frac{2 K^{2} \mathrm{i}(\lambda K-K)}{\pi(\lambda K)^{2}} J_{\alpha, \beta}, \\
& b_{1,2}=\frac{2 K^{2}}{\lambda_{1} K\left(\lambda_{1} K+K\right)} \pm \frac{2 K^{2} \mathrm{i}\left(\lambda_{1} K-K\right)}{\pi\left(\lambda_{1} K\right)^{2}} J_{1,2}, \quad b_{3,4}=\frac{2 K^{3}}{\bar{\lambda}_{1} K\left(\bar{\lambda}_{1} K+K\right)} \pm \frac{2 K^{2} \mathrm{i}\left(\bar{\lambda}_{1} K-K\right)}{\pi\left(\bar{\lambda}_{1} K\right)^{2}} J_{3,4}, \\
& I_{\alpha}=\pi\left[\frac{1}{(\lambda K)^{2}-K^{2}}+\frac{1}{2 K \Lambda_{0}(\mathrm{i} \lambda K)}\left\{\frac{\Lambda_{0}(-\mathrm{i} K)}{\lambda K+K}-\frac{\Lambda_{0}(\mathrm{i} K)}{\lambda K-K}\right\}\right], \\
& J_{\alpha}=\pi \mathrm{i}\left[\frac{\lambda K}{(\lambda K)^{2}-K^{2}}-\frac{1}{2 \Lambda_{0}(\mathrm{i} \lambda K)}\left\{\frac{\Lambda_{0}(-\mathrm{i} K)}{\lambda K+K}+\frac{\Lambda_{0}(\mathrm{i} K)}{\lambda K-K}\right\}\right] .
\end{aligned}
$$

$I_{\beta}, I_{1}, I_{2}, I_{3}, I_{4}=I_{\alpha}$ with $\lambda$ replaced by $-\lambda, \lambda_{1},-\lambda_{1}, \bar{\lambda}_{1},-\bar{\lambda}_{1}$, respectively. Similar arguments hold for the $J$ 's also.

$$
c_{\alpha}=(\lambda K)^{3}+\frac{\lambda K-K}{D(\lambda K)^{2}}, \quad c_{1}=\left(\lambda_{1} K\right)^{3}+\frac{\lambda_{1} K-K}{D\left(\lambda_{1} K\right)^{2}}, \quad c_{3}=\left(\overline{\left.\lambda_{1} K\right)^{3}}+\frac{\bar{\lambda}_{1} K-K}{D\left(\bar{\lambda}_{1} K\right)^{2}} .\right.
$$


In order to determine the first-order approximations to the constants, $B_{1}^{1}(\xi)$ and $C_{1}^{1}(\xi)$ are substituted from (3.25) and (3.26) in Equations (3.29-3.36). This gives rise to the following matrix equation

$$
\mathcal{A}^{1} X^{1}=\mathcal{B},
$$

where $\mathcal{A}^{1}=\left(a_{i j}^{1}\right)$ and $X^{1}=\left[\alpha^{1}, \beta^{1}, \ldots, R^{1}, T^{1}\right]$. The coefficients $a_{i j}^{1}$ are computed from the following:

$$
\begin{aligned}
& a_{11}^{1}=a_{32}^{1}=a_{11}^{0}-\frac{2 K^{3}(\lambda K-K)}{\pi(\lambda K)^{2}} \mathrm{e}^{\mathrm{i} \lambda K l} I_{\alpha l}, \quad a_{12}^{1}=a_{31}^{1}=a_{12}^{0}-\frac{2 K^{3}(\lambda K-K)}{\pi(\lambda K)^{2}} I_{\beta l} \\
& a_{13}^{1}=a_{34}^{1}=a_{13}^{0}-\frac{2 K^{3}\left(\lambda_{1} K-K\right)}{\pi\left(\lambda_{1} K\right)^{2}} \mathrm{e}^{\mathrm{i} \lambda_{1} K l} I_{1 l}, \quad a_{14}^{1}=a_{33}^{1}=a_{1} \mathrm{e}^{\mathrm{i} \lambda_{1} K l}-\frac{2 K^{3}\left(\lambda_{1} K-K\right)}{\pi\left(\lambda_{1} K\right)^{2}} I_{2 l} \\
& a_{15}^{1}=a_{36}^{1}=a_{4} \mathrm{e}^{-\mathrm{i} \bar{\lambda}_{1} K l}-\frac{2 K^{3}\left(\bar{\lambda}_{1} K-K\right)}{\pi\left(\bar{\lambda}_{1} K\right)^{2}} I_{3 l}, \quad a_{16}^{1}=a_{35}^{1}=a_{16}^{0}-\frac{2 K^{3}\left(\bar{\lambda}_{1} K-K\right)}{\pi\left(\bar{\lambda}_{1} K\right)^{2}} \mathrm{e}^{-\mathrm{i} \bar{\lambda}_{1} K l} I_{4 l} \\
& a_{21}^{1}=-a_{42}^{1}=a_{21}^{0}+\frac{2 K^{2} \mathrm{i}(\lambda K-K)}{\pi(\lambda K)^{2}} \mathrm{e}^{\mathrm{i} \lambda K l} J_{\alpha l}, \quad a_{22}^{1}=-a_{41}^{1}=a_{22}^{0}+\frac{2 K^{2} \mathrm{i}(\lambda K-K)}{\pi(\lambda K)^{2}} J_{\beta l} \\
& a_{23}^{1}=-a_{44}^{1}=a_{23}^{0}+\frac{2 K^{2} \mathrm{i}\left(\lambda_{1} K-K\right)}{\pi\left(\lambda_{1} K\right)^{2}} \mathrm{e}^{\mathrm{i} \lambda_{1} K l} J_{1 l}, \quad a_{24}^{1}=-a_{43}^{1}=-b_{1} \mathrm{e}^{\mathrm{i} \lambda_{1} K l}+\frac{2 K^{2} \mathrm{i}\left(\lambda_{1} K-K\right)}{\pi\left(\lambda_{1} K\right)^{2}} J_{2 l} \\
& a_{25}^{1}=-a_{46}^{1}=b_{4} \mathrm{e}^{-\mathrm{i} \bar{\lambda}_{1} K l}+\frac{2 K^{2} \mathrm{i}\left(\bar{\lambda}_{1} K-K\right)}{\pi\left(\bar{\lambda}_{1} K\right)^{2}} J_{3 l}, \quad a_{26}^{1}=-a_{45}^{1}=a_{26}^{0}+\frac{2 K^{2} \mathrm{i}\left(\bar{\lambda}_{1} K-K\right)}{\pi\left(\bar{\lambda}_{1} K\right)^{2}} \mathrm{e}^{-\mathrm{i} \bar{\lambda}_{1} K l} J_{4 l} \\
& a_{51}^{1}=a_{72}^{1}=a_{51}^{0}-\frac{\lambda K-K}{\pi D(\lambda K)^{2}} \mathrm{e}^{\mathrm{i} \lambda K l} I_{\alpha l}^{\prime}, \quad a_{52}^{1}=a_{71}^{1}=a_{52}^{0}-\frac{\lambda K-K}{\pi D(\lambda K)^{2}} I_{\beta l}^{\prime} \\
& a_{53}^{1}=a_{74}^{1}=a_{53}^{0}-\frac{\lambda_{1} K-K}{\pi D\left(\lambda_{1} K\right)^{2}} \mathrm{e}^{\mathrm{i} \lambda_{1} K l} I_{1 l}^{\prime}, \quad a_{54}^{1}=a_{73}^{1}=c_{1} \mathrm{e}^{\mathrm{i} \lambda_{1} K l}-\frac{\lambda_{1} K-K}{\pi D\left(\lambda_{1} K\right)^{2}} I_{2 l}^{\prime} \\
& a_{55}^{1}=a_{76}^{1}=c_{3} \mathrm{e}^{-\mathrm{i} \bar{\lambda}_{1} K l}-\frac{\bar{\lambda}_{1} K-K}{\pi D\left(\bar{\lambda}_{1} K\right)^{2}} I_{3 l}^{\prime}, \quad a_{56}^{1}=a_{75}^{1}=a_{56}^{0}-\frac{\bar{\lambda}_{1} K-K}{\pi D\left(\bar{\lambda}_{1} K\right)^{2}} \mathrm{e}^{-\mathrm{i} \bar{\lambda}_{1} K l} I_{4 l}^{\prime} \\
& a_{61}^{1}=-a_{82}^{1}=a_{61}^{0}+\frac{\mathrm{i}(\lambda K-K)}{\pi D(\lambda K)^{2}} \mathrm{e}^{\mathrm{i} \lambda K l} J_{\alpha l}^{\prime}, \quad a_{62}^{1}=-a_{81}^{1}=a_{62}^{0}+\frac{\mathrm{i}(\lambda K-K)}{\pi D(\lambda K)^{2}} J_{\beta l}^{\prime} \\
& a_{63}^{1}=-a_{84}^{1}=a_{63}^{0}+\frac{\mathrm{i}\left(\lambda_{1} K-K\right)}{\pi D\left(\lambda_{1} K\right)^{2}} \mathrm{e}^{\mathrm{i} \lambda_{1} K l} J_{1 l}^{\prime}, \quad a_{64}^{1}=-a_{83}^{1}=-\lambda_{1} K c_{1} \mathrm{e}^{\mathrm{i} \lambda_{1} K l}+\frac{\mathrm{i}\left(\lambda_{1} K-K\right)}{\pi D\left(\lambda_{1} K\right)^{2}} J_{2 l}^{\prime} \\
& a_{65}^{1}=-a_{86}^{1}=\bar{\lambda}_{1} K c_{3} \mathrm{e}^{-\mathrm{i} \bar{\lambda}_{1} K l}+\frac{\mathrm{i}\left(\bar{\lambda}_{1} K-K\right)}{\pi D\left(\bar{\lambda}_{1} K\right)^{2}} J_{3 l}^{\prime}, \quad a_{66}^{1}=-a_{85}^{1}=a_{66}^{0}+\frac{\mathrm{i}\left(\bar{\lambda}_{1} K-K\right)}{\pi D\left(\bar{\lambda}_{1} K\right)^{2}} \mathrm{e}^{-\mathrm{i} \bar{\lambda}_{1} K l} J_{4 l}^{\prime}
\end{aligned}
$$


where

$$
\begin{aligned}
& I_{\alpha l}=\int_{0}^{\infty} \frac{P_{\alpha}(\xi) \mathrm{e}^{-\xi l}}{\xi^{2}+K^{2}} \mathrm{~d} \xi, \quad I_{\alpha l}^{\prime}=\int_{0}^{\infty} P_{\alpha}(\xi) \mathrm{e}^{-\xi l} \mathrm{~d} \xi \\
& J_{\alpha l}=\int_{0}^{\infty} \frac{\xi P_{\alpha}(\xi) \mathrm{e}^{-\xi l}}{\xi^{2}+K^{2}} \mathrm{~d} \xi, \quad J_{\alpha l}^{\prime}=\int_{0}^{\infty} \xi P_{\alpha}(\xi) \mathrm{e}^{-\xi l} \mathrm{~d} \xi
\end{aligned}
$$

The above integrals are evaluated using Watson's lemma (cf. [15, p. 438]).

\section{References}

1. D.V. Evans and T.V. Davies, Wave ice interaction. Rep. no. 1313, Davidson Laboratory, Stevents Inst. of Tech., New Jersey, USA (1968).

2. H. Chung and C. Fox, Calculation of wave ice interaction using the Wiener-Hopf technique. New Zealand J. Math. 31 (2002) 1-18.

3. R.V. Gol'dshtein and A.V. Marchenko, The diffraction of plane gravitational waves by the edge of an icecover. PMM 53 (1989) 731-736.

4. L.A. Tkacheva, Surface wave diffraction on a floating elastic plate. Fluid Dyn. 36 (2001) 776-789.

5. L.A. Tkacheva, Scattering of surface waves by the edge of a floating elastic plate. J. Appl. Mech. \& Tech. Phys. 42 (2001) 638-646.

6. L.A. Tkacheva, Hydroelastic behaviour of a floating elastic plate in waves. J. Appl. Mech. \& Tech. Phys. 42 (2001) 991-996.

7. C. Fox and V.A. Squire, On the oblique reflection and transmission of ocean waves at shore fast sea ice. Phil. Trans. R. Soc. London A347 (1994) 185-218.

8. C.M. Linton, The finite Dock problem. ZAMP 52 (2001) 640-656.

9. C.M. Linton and H. Chung, Reflection and transmission at the ocean/sea-ice boundary. Wave Motion 38 (2003) 43-52.

10. H. Chung and C.M. Linton, Reflection and transmission across a finite gap in an infinite elastic plate on water. Q. J. Mech. Appl. Math. (2005) To appear.

11. A.J. Hermans, Interaction of free-surface waves with a floating dock. J. Engng. Math. 45 (2003) $39-53$.

12. A.J. Hermans, Interaction of free-surface waves with floating flexible strips. J. Engng. Math. 49 (2004) 133147.

13. A. Chakrabarti, On the solution of the problem of scattering of surface water waves by a sharp discontinuity in the surface boundary conditions. ANZIAM J. 42 (2000) 277-286.

14. A. Chakrabarti, On the solution of the problem of scattering of surface water waves by the edge of an ice-cover. Proc. R. Soc. London A 456 (2000) 1087-1099.

15. F. Ursell, The effect of a fixed vertical barrier on surface waves in deep water. Proc. Camb. Phil. Soc. 43 (1947) 374-382.

16. D.S. Jones, The Theory of Electromagnetism. Oxford: Pergamon Press (1964) 807 pp.

17. S.A. Gabov, A.G. Svehnikov and A.K. Shatov, Dispersion of internal waves by an obstacle floating on the boundary separating two liquids. PMM 53 (1989) 727-730.

18. M. Kanoria, B.N. Mandal and A. Chakrabarti, The Wiener-Hopf solution of a class of mixed boundary value problems arising in surface water wave phenomena. Wave Motion 29 (1999) 267-292. 